\title{
Stronger correlation between antibiotic use and the incidence of Clostridium difficile determined by culture results instead of faecal toxin detection only
}

\author{
D. Mertz • R. Frei • H. Plagge • M. Battegay • \\ A. F. Widmer
}

Received: 4 June 2010 / Accepted: 26 July 2010 /Published online: 13 August 2010

(C) Springer-Verlag 2010

\begin{abstract}
The detection of Clostridium difficile in previous studies evaluating antibiotic use as a risk factor was limited to toxin assay tests. The reported associations may have been misleading due to the low sensitivity of toxin assay tests compared to culture results. Antibiotic use and the incidence of $C$. difficile of 19 units (wards) over 5 years were analysed. Stool samples were tested for toxin A/B and cultured. The correlation of antibiotic use with the incidence of $C$. difficile determined by culture results was compared to the correlation determined by toxin assay results. Additionally, single antibiotics were analysed as risk factors. Of 5,772 faecal samples tested for $C$. difficile, 154 single-first cases were detected by the toxin assay and 251 additional single-first cases by culture. Antibiotic use was a significantly stronger risk factor in the correlation based on the culture results $\left(R^{2}=0.63\right)$ versus toxin assay results $\left(R^{2}=0.40\right)$. Multivariate analysis did not improve the correlation significantly and only the group of broad-spectrum beta-lactams was identified as an independent risk factor. The correlation between antibiotic use and $C$. difficile incidence rates significantly improves if detection is not limited to faecal toxin assays. Therefore, antibiotic pressure was previously underestimated as a risk factor.
\end{abstract}

D. Mertz $\cdot$ M. Battegay $\cdot$ A. F. Widmer $(\bowtie)$

Division of Infectious Diseases and Hospital Epidemiology,

University Hospital Basel,

Petersgraben 4,

4031 Basel, Switzerland

e-mail: widmera@uhbs.ch

R. Frei

Division of Clinical Microbiology, University Hospital Basel, Basel, Switzerland

H. Plagge

Hospital Pharmacy, University Hospital Basel,

Basel, Switzerland

\section{Introduction}

Clostridium difficile is the most common cause of nosocomial diarrhoea. Potential risk factors for the acquisition of $C$. difficile and $C$. difficile infection (CDI) include host factors, poor infection control practice [1], the use of gastric acid-suppressive agents [2] and, most importantly, antibiotic use [2-5]. Antibiotics may disrupt the host defence provided by the indigenous micro-flora of the colon and, therefore, increase the risk of CDI [5]. Historically, the antibiotics most commonly associated with CDI were clindamycin, penicillins and cephalosporins, mostly of the second and third generation [3]. More recently, quinolones have been identified as a key risk factor resulting in multiple epidemics and even ward closures [5,6]. The 2010 guidelines issued by the Society for Healthcare Epidemiology of America (SHEA) and the Infectious Diseases Society of America (IDSA) recommend to minimise antibiotic use as a preventive measure and, in particular, to restrict the use of cephalosporins and clindamycin [7].

Toxin assays detect only approximately half of the cases of $C$. difficile infections compared to toxigenic culture, as previously reported in our institution [8]. Therefore, toxigenic culture, i.e. the detection of toxin $\mathrm{A} / \mathrm{B}$ in the $C$. difficile culture, has recently been described as the gold standard to diagnose CDI [7, 9]. However, studies reporting on an association between antibiotic use and the incidence of $C$. difficile were based on toxin assay results from faecal samples $[2-4,10]$. Due to the lack of sensitivity of toxin assays, the reported association might have been underestimated and misleading.

We aimed to correlate the unit-specific antibiotic use with the incidence of $C$. difficile overall, i.e. faecal toxin detection and/or positive $C$. difficile culture. We hypothesised that the inclusion of culture results may strengthen 
the correlation with antibiotic use due to the higher reliability of detection and that specific classes of antimicrobial agents are more significant predictors for colonisation or infection with $C$. difficile.

\section{Materials and methods}

\section{Setting}

The University Hospital Basel is a tertiary care centre serving 750 beds and about 30,000 admissions per year. Nineteen units were included in the study (two intensive care units, one isolation unit, five medical units, eight surgical units, one neurology unit, one otorhinolaryngology unit and one dermatology unit). Insufficient data was available from the gynaecology and obstetrics units, which were excluded.

\section{Microbiology data}

The results of faecal specimens from adult patients submitted to the laboratory for $C$. difficile testing over 5 years (January 2004 to December 2008) were prospectively collected. The microbiological tests are described elsewhere in detail [8]. In short, samples were initially tested for toxin A/B (CDIFF TOX A/B II; TechLab/ Wampole, Blacksburg, VA, USA) and routinely cultured for $C$. difficile (selective cycloserine-cefoxitin-fructose agar plates).

\section{Pharmacy data}

Antibiotic use was collected in defined daily doses (DDD) as recommended by the World Health Organization (WHO) since 2003.

\section{Statistical analysis}

In order not to violate the assumption of independence of the single observation, only the first isolate per patient and year was taken into account to calculate the incidence densities of $C$. difficile per 1,000 patient days. The rates determined by toxin assay alone were compared to the overall rates, which were based on either a positive $C$. difficile culture and/or toxin A/B assay test. The data of 19 units over 5 years allowed the correlation of 95 data points in ordinary linear regression. Single-sample $z$-statistics was performed for the comparison of correlation coefficients. Because metronidazole and vancomycin use rather represents the treatment of CDI than being a potential risk factor, the DDDs of these antibiotics were subtracted from the overall antibiotic use.
Next, simple linear regression was done for each antibiotic separately. Furthermore, single-factor and multivariate analysis of six groups of antibiotics was performed: aminoglycosides, quinolones, macrolides/lincosamides, first- to third-generation cephalosporins, broad-spectrum beta-lactams (i.e. piperacillin/tazobactam, meropenem, imipenem, cefepime) and other beta-lactams.

Additionally, a secondary analysis was completed using a generalised estimating equation approach on a negative binomial distribution correcting for repeated measurement/ auto-correlation and for the count nature of the incidence data.

A $p$-value of $\leq 0.05$ was defined as being statistically significant. The results were analysed using commercially available statistical software (SPSS 17.0; SPSS Inc., Chicago, IL, USA).

\section{Results}

Of 5,772 faecal samples tested during the 5-year study period, 154 single-first cases of $C$. difficile were detected by the toxin assay alone. In addition, 251 single-first cases of $C$. difficile were detected by positive $C$. difficile culture results (405 cases in total). The corresponding incidence rates were 0.14 and 0.38 per 1,000 patient days, respectively.

Antibiotic use correlates significantly with the incidence of C. difficile in both analyses ( $p<0.001$, Fig. 1). Importantly, the correlation is significantly better using culture results than toxin assay results only $\left(R^{2}=0.632\right.$ vs. $R^{2}=0.397$; $p<0.001)$.

Several antibiotics were identified as risk factors for a higher incidence of $C$. difficile overall in the single-factor analysis: piperacillin/tazobactam $(R=0.85)$, trimethoprim/ sulfamethoxazole (TMP/SMX; $R=0.80)$, cefepime $(R=$ $0.75)$, meropenem $(R=0.81)$, imipenem/cilastatin $(R=$ $0.38)$, levofloxacin $(R=0.50)$ and tetracyclines $(R=0.23)$. Notably, all other antibiotics, including clindamycin and quinolones other than levofloxacin, were not associated risk factors. Grouping the antibiotics, the group of broadspectrum beta-lactams $(R=0.84)$, aminoglycosides $(R=$ $0.81)$ and the group of macrolides/lincosamides $(R=0.29)$ were identified as significant risk factors in the single-factor analysis. In multivariate analysis, only the groups of macrolides/lincosamides and broad-spectrum beta-lactams remained significant independent risk factors for both outcomes, i.e. the incidence of $C$. difficile overall and the incidence determined by toxin assay results only. However, the goodness of fit of the multivariate model $\left(R^{2}=0.762\right.$ and 0.457 , respectively) was not significantly better than for the model with antibiotic use overall as the only predictor. Importantly, the group of quinolones was not significantly associated with the incidence of $C$. difficile in either model. 


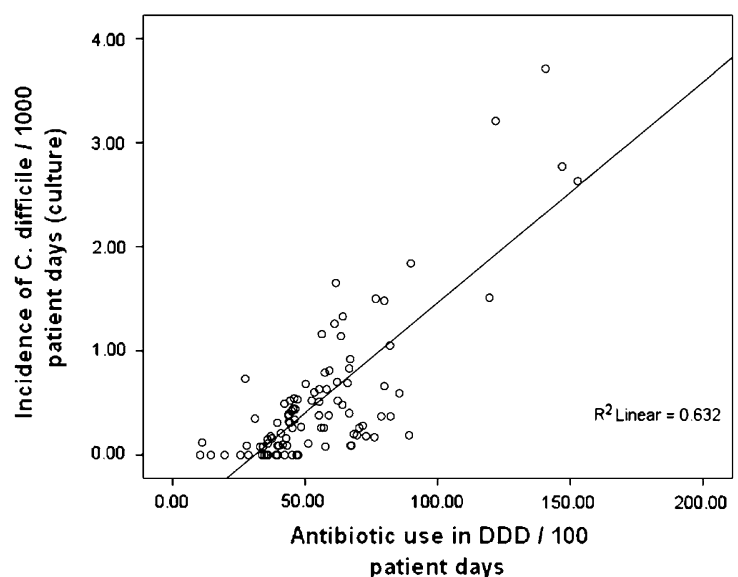

Fig. 1 Correlation between unit-specific antibiotic use and the incidence of Clostridium difficile. Every dot represents one of 95 observations (annual data over 5 years from 19 hospital units). The

\section{Discussion}

The correlation between antibiotic use and $C$. difficile incidence significantly improves if detection includes culture results. This study provides new insights in the correlation of antibiotic use and the incidence of $C$. difficile, and has important implications for research and the (inappropriate) use of antibiotics: antibiotics as a risk factor were underestimated in the past and, for future research, cultures should be used to obtain a more appropriate estimate of antibiotic use as a risk factor for $C$. difficile. In addition, the effect of infection control practices may have been overestimated, since antibiotic use may be more important than previously thought.

The incidence determined by the results of culture consists of approximately $40 \%$ of cases also detected by the toxin assay, $40 \%$ of cases with toxigenic strains undetected by the toxin assay and $20 \%$ of cases with non-toxigenic strains [8]. Therefore, the increase in the correlation is based on both the additional number of cases infected by toxigenic strains and a smaller number of cases colonised by non-toxigenic strains. Antibiotic pressure selects for $C$. difficile but is not restricted to toxin A/B-positive strains. Getting colonised is a consequence of the predicting factors, but whether one gets colonised by a toxin-producing or a non-toxigenic strain might be just a matter of chance and may have further biased the results in previous studies.

Antibiotics as risk factors were likely underestimated in studies in the past due to the restriction of detection to toxin assays, explaining the lower level of correlation in previous studies $[3,4,10]$ or the need to use very sophisticated models to be able to show similarly strong correlations $[2,4]$. The data shows that a simple model explains more than $60 \%$ of the variance of the incidence of $C$. difficile using the overall antibiotic use as a single risk factor. In the single-factor

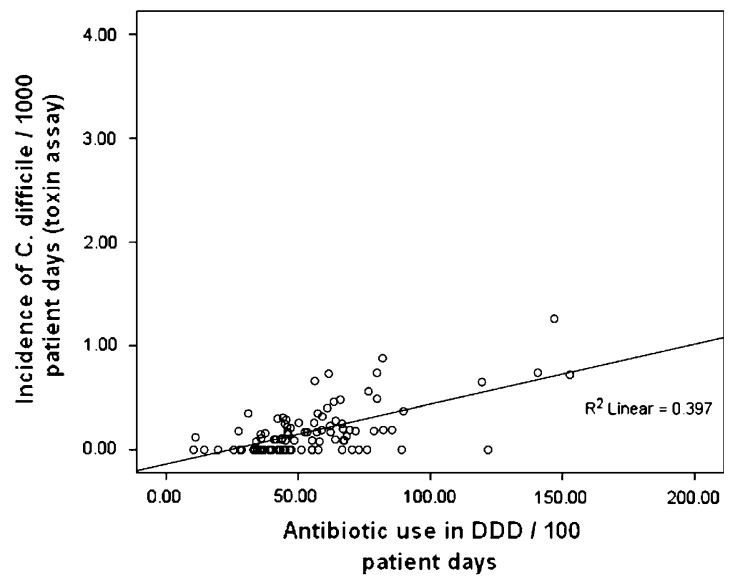

straight line represents the line of best fit of the ordinary linear regression analysis. Antibiotic use refers to the cumulative use of all antibiotics, with the exception of vancomycin and metronidazole

analysis, the use of certain broad-spectrum beta-lactams, i.e. piperacillin/tazobactam, cefepime and meropenem, as well as $\mathrm{TMP} / \mathrm{SMX}$ and the aminoglycosides, each explained more than $60 \%$ of the variance of $C$. difficile incidence. In multivariate analysis, only the groups of macrolides/lincosamides and, most importantly, broad-spectrum beta-lactams were found to be independent risk factors. In the secondary analysis, only the latter group remained as an independent risk factor. Importantly, the predictions in the multivariate analysis were not significantly better than the prediction based on the overall antibiotic use as the only risk factor.

Unexpectedly, the association with quinolone use was not observed in contrast to other reports, e.g. by Pépin [6]. One may hypothesise that quinolones select predominantly for quinolone-resistant strains such as NAP1/027 and, consequently, that the lack of a significant association in this study is due to the comparable low incidence of quinolone-resistant strains $(12.2 \%$ of the last 172 isolates with $\mathrm{MHK} \geq 8 \mathrm{mg} / \mathrm{L}$ ). Another explanation may be the fact that the low use of quinolones in our hospital of around 6 DDD/100 patient days may not be sufficient to induce a higher incidence of $C$. difficile.

This study adds significantly to previous data limited to faecal toxin-positive samples showing a correlation between antibiotic use and the incidence of $C$. difficile. For the sake of simplicity, the results of the ordinary linear regression were reported. Importantly, our secondary analysis taking into account repeated measurements and the count nature of the incidence data corroborated the findings. There are some limitations to the study. First, the association between antibiotic use with $C$. difficile rather than with $\mathrm{CDI}$ was estimated. However, according to our hospital policy, only loose stool specimens are to be sent to the laboratory for testing for $C$. difficile. Second, the multivariate analysis should be interpreted with caution due to the considerably 
low number of observations. Third, the findings are based on aggregate data, i.e. on the unit level, and caution is warranted if generalising to the individual level.

In conclusion, the correlation between antibiotic use and C. difficile incidence rates significantly improves if detection includes culture results and is not limited to faecal toxin assays. Hence, antibiotic pressure as a risk factor was underestimated in previous studies. The results further underline the importance of the overall pressure of antibiotics that explains the majority of the variance of $C$. difficile incidence rates rather than the importance of a single group of antimicrobial agents such as quinolones, which were not significantly associated. Therefore, interventions to reduce the overall antibiotic use might be more successful in controlling $C$. difficile incidence than interventions focusing on certain groups of antibiotics.

Financial support The study was supported by an unrestricted grant from the "Stiftung Forschung Infektionskrankheiten" (SFI).

Transparency declaration The authors have no conflicts of interests to declare.

Previous presentation An abstract was presented at the 20th European Congress of Clinical Microbiology and Infectious Diseases (ECCMID), Vienna, Austria, April 2010 (Abstract O-161).

\section{References}

1. McFarland LV (2008) Update on the changing epidemiology of Clostridium difficile-associated disease. Nat Clin Pract Gastroenterol Hepatol 5(1):40-48
2. Aldeyab MA, Harbarth S, Vernaz N, Kearney MP, Scott MG, Funston C, Savage K, Kelly D, Aldiab MA, McElnay JC (2009) Quasiexperimental study of the effects of antibiotic use, gastric acid-suppressive agents, and infection control practices on the incidence of Clostridium difficile-associated diarrhea in hospitalized patients. Antimicrob Agents Chemother 53 (5):2082-2088

3. Thomas C, Stevenson M, Riley TV (2003) Antibiotics and hospital-acquired Clostridium difficile-associated diarrhoea: a systematic review. J Antimicrob Chemother 51(6):1339-1350

4. Vernaz N, Hill K, Leggeat S, Nathwani D, Philips G, Bonnabry P, Davey P (2009) Temporal effects of antibiotic use and Clostridium difficile infections. J Antimicrob Chemother 63 (6):1272-1275

5. Owens RC Jr, Donskey CJ, Gaynes RP, Loo VG, Muto CA (2008) Antimicrobial-associated risk factors for Clostridium difficile infection. Clin Infect Dis 46(Suppl 1):S19-S31

6. Pépin J, Saheb N, Coulombe MA, Alary ME, Corriveau MP, Authier S, Leblanc M, Rivard G, Bettez M, Primeau V, Nguyen M, Jacob CE, Lanthier L (2005) Emergence of fluoroquinolones as the predominant risk factor for Clostridium difficile-associated diarrhea: a cohort study during an epidemic in Quebec. Clin Infect Dis 41(9): 1254-1260

7. Cohen SH, Gerding DN, Johnson S, Kelly CP, Loo VG, McDonald LC, Pépin J, Wilcox MH, Society for Healthcare Epidemiology of America; Infectious Diseases Society of America (2010) Clinical practice guidelines for Clostridium difficile infection in adults: 2010 update by the society for healthcare epidemiology of America (SHEA) and the infectious diseases society of America (IDSA). Infect Control Hosp Epidemiol 31 (5):431-455

8. Fenner L, Widmer AF, Goy G, Rudin S, Frei R (2008) Rapid and reliable diagnostic algorithm for detection of Clostridium difficile. J Clin Microbiol 46(1):328-330

9. Crobach MJ, Dekkers OM, Wilcox MH, Kuijper EJ (2009) European Society of Clinical Microbiology and Infectious Diseases (ESCMID): data review and recommendations for diagnosing Clostridium difficile-infection (CDI). Clin Microbiol Infect 15(12):1053-1066

10. Vernaz N, Sax H, Pittet D, Bonnabry P, Schrenzel J, Harbarth S (2008) Temporal effects of antibiotic use and hand rub consumption on the incidence of MRSA and Clostridium difficile. J Antimicrob Chemother 62(3):601-607 